\title{
Novel Risk Score Efficiently Prevents Tolvaptan-Induced Hypernatremic Events in Patients With Heart Failure
}

\author{
Koichiro Kinugawa, MD, PhD; Naoki Sato, MD, PhD; Takayuki Inomata, MD, PhD; \\ Moriyoshi Yasuda, PhD; Yoshiyuki Shibasaki, BSc; Toshiyuki Shimakawa, BSc
}

\begin{abstract}
Background: It has been 7 years since tolvaptan was approved in Japan for the indication of heart failure in patients with volume overload; the drug can be used in patients with normonatremia. Hypernatremia was identified as a significant adverse event to be prevented.
\end{abstract}

\begin{abstract}
Methods and Results: We compiled and analyzed data from 3,349 patients over 5 years to identify patients at high risk of hypernatremia with tolvaptan treatment. The incidence of hypernatremia, defined as serum sodium $\geq 150 \mathrm{mEq} / \mathrm{L}$, was $3.65 \%$. Baseline serum sodium concentrations, serum potassium concentrations, blood urea nitrogen : creatinine ratio, initial tolvaptan dose, and age were identified as risk factors for hypernatremia. A hypernatremia risk score was developed using the odds ratios for these factors. The high-risk population was defined as patients with a risk score $\geq 17.80$.
\end{abstract}

Conclusions: To prevent the occurrence of hypernatremic events in patients taking tolvaptan, we recommend a very low starting dose (i.e., $3.75 \mathrm{mg} /$ day) in patients identified as being at high risk of hypernatremia using our new scoring process.

Key Words: Aquauretic; Diuretics; Hypernatremia

D iuretic resistance was common in the Acute Decompensated Heart Failure Registry (ADHERE), ${ }^{1}$ being observed in $30 \%$ of patients with heart failure. Until the approval of tolvaptan, a vasopressin $\mathrm{V}_{2}$ receptor antagonist, there were limited therapeutic options for patients with heart failure and fluid retention who did not respond sufficiently to conventional diuretics.

Tolvaptan has been approved in the US since 2009, with current indications for tolvaptan being the treatment of clinically significant hypervolemic and euvolemic hyponatremia, as well as Syndrome of Inappropriate Antidiuretic Hormone (SIADH). ${ }^{2}$ However, in Japan, tolvaptan was approved in 2010 for the following expanded indication, which is quite different from indications in the US: "volume overload in heart failure patients when adequate response was not obtained with other diuretics (e.g., loop diuretics)" ${ }^{3,4}$ Tolvaptan significantly increases urine volume with water excretion into the urine $e^{5}$ and sometimes results in marked increases in serum sodium concentrations. Therefore, tolvaptan treatment must be started in an inpatient setting to avoid the adverse event of hypernatremia caused by its pharmacological action of aquaresis. In the EVEREST study, in which patients were treated with $30 \mathrm{mg}$ tolvaptan, the incidence of hypernatremia was $1.7 \% .^{6}$ In another study, abnormally high serum sodium concentra- tions were reported to occur following tolvaptan treatment in a dose-dependent manner, with rates of $6 \%, 11 \%$, and $13 \%$ for 30,45 , and $60 \mathrm{mg}$ tolvaptan, respectively. ${ }^{7}$

Although the risk of hypernatremia was not clear in the pivotal Phase 3 QUEST study, ${ }^{8}$ we reported 3 risk factors for hypernatremic events using the interim results of the Samsca Post-Marketing Surveillance In the Heart faiLurE (SMILE) study conducted in 2014. ${ }^{9}$ These factors were higher baseline sodium concentration, lower baseline potassium concentration, and a $15-\mathrm{mg}$ starting dose of tolvaptan. Moreover, based on subanalysis of the SMILE study in $2015,{ }^{10}$ we recommended a lower dose of tolvaptan for older patients (i.e., those $>80$ years of age) to prevent hypernatremic events. Herein, we report on the development of a risk score to predict the prevalence of hypernatremia using the final dataset of the SMILE study.

\section{Methods}

The present prospective multicenter observational study was conducted in compliance with Good Post-Marketing Study Practice, an ordinance issued by the Ministry of Health, Labor and Welfare for the implementation of post-marketing surveillance of new drugs approved in Japan. The study period was from 2011 to 2016, and the

Received October 10, 2017; revised manuscript received January 18, 2018; accepted January 29, 2018; released online March 31, 2018 Time for primary review: 27 days

Second Department of Internal Medicine, University of Toyama, Toyama (K.K.); Division of Cardiology and Intensive Care Unit, Nippon Medical School, Musashi-Kosugi Hospital, Kawasaki (N.S.); Department of Cardiovascular Medicine, Kitasato University, Kitasato Institute Hospital, Tokyo (T.I.); and Otsuka Pharmaceutical Co. Ltd., Tokyo (M.Y., Y.S., T.S.), Japan

Mailing address: Koichiro Kinugawa, Professor, MD, PhD, Second Department of Internal Medicine, University of Toyama, 2630 Sugitani, Toyama 930-0914, Japan. E-mail: kinugawa0422@gmail.com

ISSN-1346-9843 All rights are reserved to the Japanese Circulation Society. For permissions, please e-mail: cj@j-circ.or.jp 


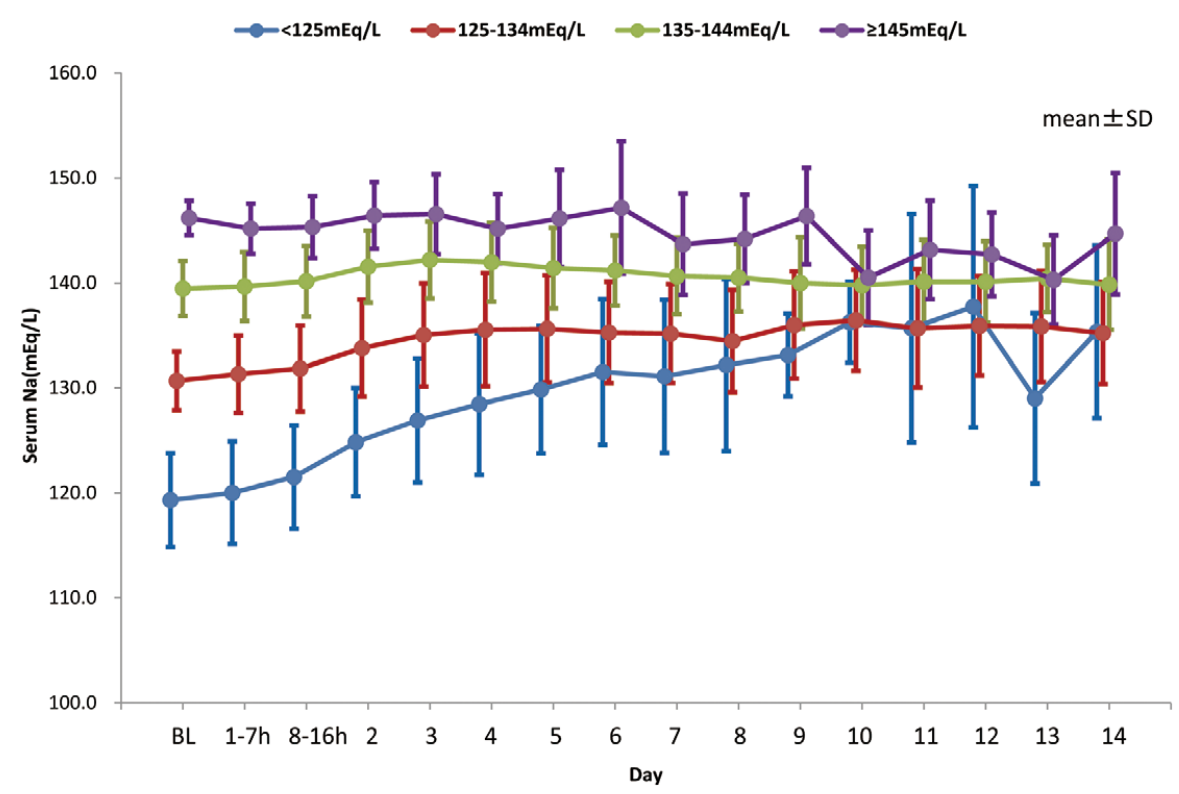

Figure 1. Changes in serum sodium concentrations from baseline $(B L)$ to Day 14 , stratified according to baseline sodium concentrations, in patients taking tolvaptan. Data are the mean \pm SD.

target number of patients was 3,000. Because the present study was a naturalistic, non-interventional, observational survey involving the collection of anonymous data from clinical settings, obtaining informed consent from patients and approval by the institutional review board of medical institutions were not mandatory, but both were obtained according to the regulations of each medical institution. This approach was fully compliant with Japanese regulations for post-marketing surveillance. In all, 492 hospitals across Japan participated in the present study.

\section{Target Patients}

The study enrolled patients with heart failure and volume overload who were resistant to loop diuretics, excluding those who had anuria, disturbed consciousness, difficulties with water intake, or hypernatremia, or who were pregnant. The standard observation period was specified as 2 weeks, but some patients were observed for longer than 2 weeks at the discretion of attending physicians.

\section{Safety Evaluation}

The laboratory parameters examined were serum sodium and potassium concentrations, alanine aminotransferase (ALT), aspartate aminotransferase (AST), total bilirubin, blood urea nitrogen (BUN), creatinine $(\mathrm{Cr})$, estimated glomerular filtration rate (eGFR), and albumin. Clinically significant events were defined as death, prolongation of hospital stay, and life-threatening events as determined by a physician. The all-cause mortality rate was calculated based on the number of deaths reported as outcomes of adverse drug events.

\section{Statistical Analysis}

In analyses of data about hypernatremia, a patient developing hypernatremia was defined as a patient whose serum sodium concentration was $<150 \mathrm{mEq} / \mathrm{L}$ at baseline and increased to $\geq 150 \mathrm{mEq} / \mathrm{L}$ after treatment. The criterion of $150 \mathrm{mEq} / \mathrm{L}$ was set in reference to Common Terminology Criteria for Adverse Events (CTCAE):11 $150 \mathrm{mEq} / \mathrm{L}$ is the cut-off point for Grade 2 in hypernatremia. In the analysis of hypernatremia, 2,875 patients for whom data on serum concentrations were available at baseline and for at least 1 post-baseline measurement were included. Risk factor analyses began with a univariate analysis in which baseline characteristics were compared between patients developing and not developing hypernatremia to determine statistically significant $(\mathrm{P}<0.05)$ parameters. Continuous variables are given as the mean $\pm \mathrm{SD}$ and categorical valuables are given as percentages. Data were analyzed using Student's t-test, Fisher's exact test, or the Chi-squared test as appropriate. Statistically significant $(\mathrm{P}<0.05)$ parameters were selected from univariate analysis, and logistic regression analyses were performed using the selected parameters. Odds ratios (ORs) and 95\% confidence intervals (CIs) were calculated and age, although not a statistically significant parameter, was also used as a risk factor for hypernatremia, taking into consideration our previous finding ${ }^{10}$ that older patients are prone to hypernatremia. A hypernatremia risk score was then derived based on the ORs for each factor. The cut-off point was calculated using receiver operating characteristic (ROC) curve analysis. Internal validation was confirmed by dividing the entire cohort into derivation and validation cohorts (patients allocated to the 2 cohorts in a ratio of 60/40 and 50/50, creating 2 different subanalysis sets). The bootstrap method (500 replications) was used to validate the modeling process by extracting $70 \%$ of data $(n=2,340)$ to derive $95 \%$ CIs. Kaplan-Meier analysis was performed to determine hypernatremic event-free rates. Although the standard observational period was set as 2 weeks, $44 \%$ of patients continued tolvaptan treatment. Analyses of hypernatremia after 2 weeks treatment are also appropriate. All these analyses were performed using SAS 


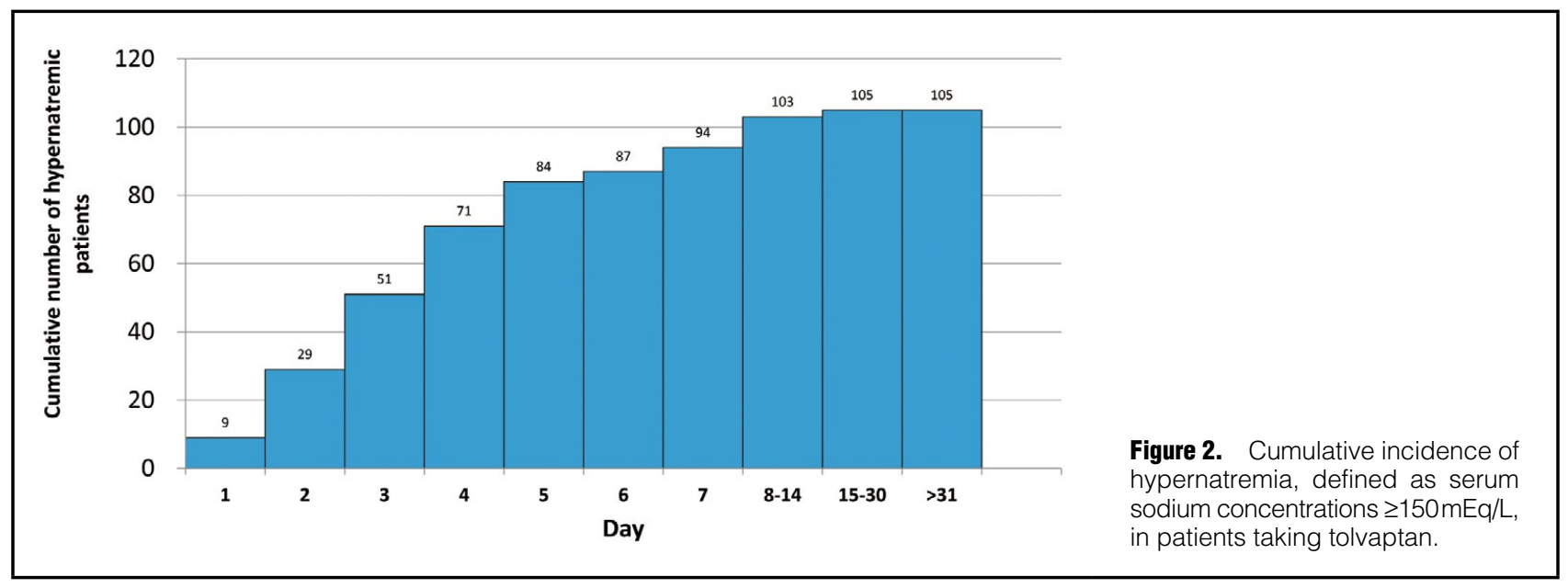

version 9.4 and JMP 13 (SAS Institute, Cary, NC, USA).

\section{Results}

\section{Patients' Characteristics}

The severity of the heart failure of enrolled patients was investigated using B-type natriuretic peptide (BNP), $\mathrm{N}$-terminal pro B-type natriuretic peptide (NT-proBNP), and the New York Heart Association (NYHA) classification. The mean and median BNP concentrations were $1,035 \pm 1,643$ and $668 \mathrm{pg} / \mathrm{mL}$, respectively $(\mathrm{n}=1,250)$, whereas the mean and median NT-proBNP concentrations were $8,313 \pm 11,176$ and $4,680 \mathrm{pg} / \mathrm{mL}(\mathrm{n}=219)$, respectively. The proportion of patients classified as NYHA Class 1, 2, 3 and 4 was $5.3 \%, 22.3 \%, 41.6$, and $20.2 \%$, respectively; NYHA classification was unknown in $10.6 \%$ of patients.

\section{Baseline Serum Sodium Concentrations and Dose of Tolvaptan}

In all, 3,350 patients were registered for the study. Of these, 3,349 were included in the analysis population, with 1 patient who did not receive tolvaptan being excluded. The mean baseline serum sodium concentration was $136.9 \pm 6.2 \mathrm{mEq} / \mathrm{L}$, with $4.5 \%, 23.2 \%, 66.8 \%$, and $5.6 \%$ of patients having mean baseline serum sodium concentrations $<125$, between $\geq 125$ and $<135$, between $\geq 135$ and $<145$, and $\geq 145 \mathrm{mEq} / \mathrm{L}$, respectively. The mean baseline daily dose of a loop diuretic was $62.3 \pm 71.3 \mathrm{mg}$, with a median dose of $40 \mathrm{mg}$. Doses of loop diuretics are shown as furosemide equivalents. The proportion of patients with doses of loop diuretics of $<40,40-79,80-119$, and $\geq 120 \mathrm{mg}$ was $26.0 \%, 44.4 \%, 18.1 \%$, and $11.5 \%$, respectively.

The mean daily dose of tolvaptan in the overall study period was $9.4 \pm 3.8 \mathrm{mg} /$ day (median $7.5 \mathrm{mg} /$ day). The proportion of patients with an initial dose of tolvaptan of 3.75 , 7.5 , and $15 \mathrm{mg}$ was $12.2 \%, 62.0 \%$, and $25.5 \%$, respectively.

\section{Changes in Serum Sodium Concentrations}

Figure 1 shows the time course of mean serum sodium concentrations in patients categorized into 4 groups based on baseline sodium concentrations. Mean serum sodium concentrations increased from baseline to Day 7 in the $<125 \mathrm{mEq} / \mathrm{L}$ group (from 119.3 to $131.1 \mathrm{mEq} / \mathrm{L}$ ) and in the $<135 \mathrm{mEq} / \mathrm{L}$ group (from 130.7 to $135.2 \mathrm{mEq} / \mathrm{L}$ ), but remained nearly unchanged in the $\geq 135 \mathrm{mEq} / \mathrm{L}$ group (from
139.5 to $140.6 \mathrm{mEq} / \mathrm{L}$ ) and $\geq 145 \mathrm{mEq} / \mathrm{L}$ group (from 146.2 to $143.7 \mathrm{mEq} / \mathrm{L}$ ).

\section{Incidence of Hypernatremia and All-Cause Mortality}

Of 2,875 patients for whom baseline and post-treatment serum sodium data were available, $105(3.65 \%)$ developed hypernatremia, as above defined. Figure 2 shows that $90 \%$ of the cases of hypernatremia occurred within 7 days of the start of treatment. These results suggest that the initial dose of tolvaptan may affect the incidence of hypernatremic events. The incidence rate of clinically significant hypernatremia, as assessed by the attending physician, was $0.42 \%$ $(14 / 3,349)$. The details of these 14 patients are given in Table S1. Among these 14 patients, 10 patients were administered $15 \mathrm{mg}$ tolvaptan and 4 patients received $7.5 \mathrm{mg}$ tolvaptan. We thought it difficult to reject a relationship between tolvaptan and hypernatremia considering the pharmacological action of tolvaptan, even if physicians ruled out a relationship with tolvaptan. Among the 14 patients with clinically significant hypernatremia, 7 deaths were reported. In the case of 5 of these deaths, the cause of death was reported as liver failure, liver cirrhosis, disseminated intravascular coagulation, and pneumonia, and these causes are less likely to be related to hypernatremia. However, in the case of the other 2 deaths, there could be a relationship with hypernatremia, because the cause of death was reported as serious heart failure and multiple organ dysfunction.

The all-cause mortality rate was $4.2 \%(139 / 3,349)$. The most frequently reported cause of death was worsening of heart failure, in approximately $44 \%$ of patients.

\section{Risk Factor Analysis for Hypernatremia}

The baseline characteristics of the 105 patients who developed hypernatremia and the 2,770 patients who did not were compared to analyze risk factors for hypernatremia (Table 1). Statistically significant $(\mathrm{P}<0.05)$ parameters were age, serum $\mathrm{Na}$, serum $\mathrm{K}, \mathrm{BUN} / \mathrm{Cr}$ ratio, albumin, and the use of $\beta$-blockers. In addition to baseline characteristics, the daily dose of loop diuretics (furosemide equivalent) and the dose of tolvaptan were compared. There was no significant difference in the dose of loop diuretics between those who did and did not develop hypernatremia $(70.8$ vs. $63.4 \mathrm{mg}$, respectively; $\mathrm{P}=0.4012$ ), but the dose of tolvaptan was significantly higher in patients who developed 


\begin{tabular}{|c|c|c|c|}
\hline & \multicolumn{2}{|c|}{ Hypernatremic event } & \multirow{2}{*}{$P$ value } \\
\hline & Yes & No & \\
\hline Age (years) & $80.1 \pm 11.5$ & $77.2 \pm 12.4$ & $0.0171^{A}$ \\
\hline$\%$ Female & 44.8 & 43.4 & $0.8412^{B}$ \\
\hline Body weight (kg) & $55.2 \pm 12.7$ & $57.8 \pm 14.2$ & $0.2278^{\mathrm{A}}$ \\
\hline NYHA class (\%) & & & $0.5658^{\mathrm{B}}$ \\
\hline 1 or 2 & 23.8 & 26.4 & \\
\hline 3 or 4 & 65.7 & 61.8 & \\
\hline Unknown & 10.5 & 11.8 & \\
\hline Type of HF (\%) & & & $0.1761^{\mathrm{C}}$ \\
\hline Left HF & 25.7 & 32.4 & \\
\hline Right HF & 8.6 & 11.3 & \\
\hline Both right and left HF & 63.8 & 53.1 & \\
\hline Unknown & 1.9 & 2.6 & \\
\hline Others & 0.0 & 0.7 & \\
\hline Cardiomyopathy/myocarditis (\%) & 14.3 & 17.0 & $0.5923^{\mathrm{B}}$ \\
\hline Angina/MI (\%) & 34.3 & 38.6 & $0.4675^{\mathrm{B}}$ \\
\hline Valvular disease (\%) & 27.6 & 33.0 & $0.3332^{\mathrm{B}}$ \\
\hline Hypertensive (\%) & 24.8 & 20.8 & $0.3220^{B}$ \\
\hline Arrhythmia (\%) & 27.6 & 27.2 & $0.8220^{B}$ \\
\hline $\mathrm{Na}(\mathrm{mEq} / \mathrm{L})$ & $140.4 \pm 7.6$ & $136.8 \pm 6.2$ & $<0.0001^{\mathrm{A}}$ \\
\hline $\mathrm{K}(\mathrm{mEq} / \mathrm{L})$ & $3.9 \pm 0.8$ & $4.2 \pm 0.6$ & $0.0022^{\mathrm{A}}$ \\
\hline BUN (mg/dL) & $36.1 \pm 23.5$ & $33.5 \pm 20.5$ & $0.2133^{A}$ \\
\hline $\mathrm{Cr}(\mathrm{mg} / \mathrm{dL})$ & $1.55 \pm 1.15$ & $1.56 \pm 1.06$ & $0.9176^{\mathrm{A}}$ \\
\hline $\mathrm{BUN} / \mathrm{Cr}$ & $26.3 \pm 13.6$ & $23.3 \pm 10.0$ & $0.0028^{A}$ \\
\hline eGFR (mL/min/1.73 m²) & $44.7 \pm 26.1$ & $43.4 \pm 26.8$ & $0.6240^{\mathrm{A}}$ \\
\hline Albumin (g/dL) & $3.0 \pm 0.5$ & $3.2 \pm 0.6$ & $0.0335^{\mathrm{A}}$ \\
\hline AST (IU/L) & $68 \pm 123$ & $47 \pm 248$ & $0.4460^{\mathrm{A}}$ \\
\hline ALT (IU/L) & $56 \pm 116$ & $39 \pm 240$ & $0.5326^{A}$ \\
\hline 24-h urine volume $(\mathrm{mL})$ & $1,378 \pm 1,095$ & $1,368 \pm 827$ & $0.9328^{A}$ \\
\hline $\mathrm{SBP}(\mathrm{mmHg})$ & $122.1 \pm 22.6$ & $118.7 \pm 22.4$ & $0.1971^{\mathrm{A}}$ \\
\hline $\mathrm{DBP}(\mathrm{mmHg})$ & $65.0 \pm 13.9$ & $65.9 \pm 13.9$ & $0.5537^{\mathrm{A}}$ \\
\hline \multicolumn{4}{|l|}{ Medications } \\
\hline ACEI (\%) & 21.0 & 17.0 & $0.2917^{B}$ \\
\hline ARB (\%) & 18.1 & 26.6 & $0.0548^{B}$ \\
\hline$\beta$-blocker (\%) & 30.5 & 40.9 & $0.0336^{B}$ \\
\hline Thiazide (\%) & 4.8 & 7.6 & $0.3472^{B}$ \\
\hline Aldosterone antagonist (\%) & 36.2 & 37.2 & $0.9181^{B}$ \\
\hline Carperitide (\%) & 19.1 & 17.3 & $0.6022^{\mathrm{B}}$ \\
\hline
\end{tabular}

Unless indicated otherwise, data are given as the mean \pm SD. AStudent's t-test; ${ }^{B}$ Fisher's exact test; ${ }^{\circ}$ Chi-squared test. ACEI, angiotensin-converting enzyme inhibitor; ALT, alanine aminotransferase; ARB, angiotensin receptor blocker; AST, aspartate aminotransferase; BUN, blood urea nitrogen; $\mathrm{Cr}$, creatinine; DBP, diastolic blood pressure; eGFR, estimated glomerular filtration rate; HF, heart failure; MI, myocardial infarction; NYHA, New York Heart Association; SBP, systolic blood pressure.

hypernatremia (10.7 vs. $8.8 \mathrm{mg} ; \mathrm{P}<0.0001)$.

Table 2 shows ORs and B values (log-transformed ORs). Only age was not statistically significant in the multiple regression analysis for factors predicting hypernatremia. An equation for calculating a risk score using the B values was then created, as shown in Figure 3. Age was included in this equation even though it was not a statistically significant parameter, because in a previous study ${ }^{10}$ we found that older patients ( $\geq 80$ years of age) are prone to hypernatremia. The risk scores calculated were normally distributed. The mean risk score was 17.24 \pm 0.93 (Figure 3A). An ROC analysis was performed to determine the cut-off value needed to obtain the highest area under the curve (AUC) to set the hypernatremia as an objective variable. The cut-off value was determined to be 17.80 when the AUC was 0.735 . Based on risk scores, patients were divided into 2 groups (risk scores $<17.80$ or $\geq 17.80$ ), and a KaplanMeier analysis was performed on hypernatremia event-free rates (Figure 3B). Furthermore, each of the 2 main groups was divided into 3 subgroups based on the initial dose of tolvaptan (3.75, 7.5, or $15 \mathrm{mg}$; Figure 4). The incidence of hypernatremia was low in patients with a risk score $<17.80$, including those receiving an initial dose of $15 \mathrm{mg}$ tolvaptan (Figure 4A). Meanwhile, among patients with a risk score of $\geq 17.80$, the incidence of hypernatremia became increased dose dependently in patients receiving initial doses of 3.75 , 


\begin{tabular}{|lcccc|}
\multicolumn{4}{l}{ Table 2. Multiple Regression Model for Predicting } & \multicolumn{3}{l|}{ Hypernatremic Events } & With Continuous Variables \\
Serum Na & OR & B (=log OR) & $95 \%$ Cl & P value \\
BUN/Cr & 1.133 & 0.125 & $1.079-1.19$ & $<0.0001$ \\
Serum K & 1.033 & 0.032 & $1.017-1.049$ & $<0.0001$ \\
Age & 0.646 & -0.436 & $0.453-0.922$ & 0.0161 \\
\hline
\end{tabular}

BUN, blood urea nitrogen; $\mathrm{Cl}$, confidence interval; $\mathrm{Cr}$, creatinine; OR, odds ratio.

\section{Risk score $=0.125 x(\mathrm{Na})+0.032 x(\mathrm{BUN} / \mathrm{Cr})-0.436 x(\mathrm{~K})+0.014 \times(\mathrm{Age})$}

(A)

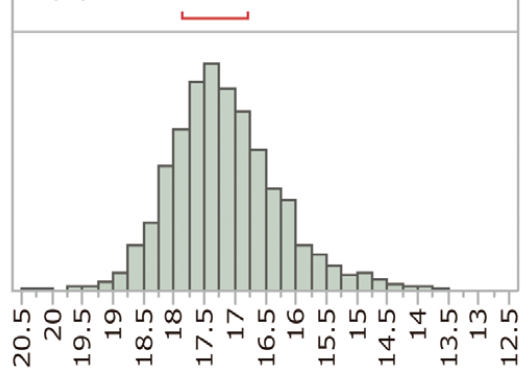

Risk score
(B)

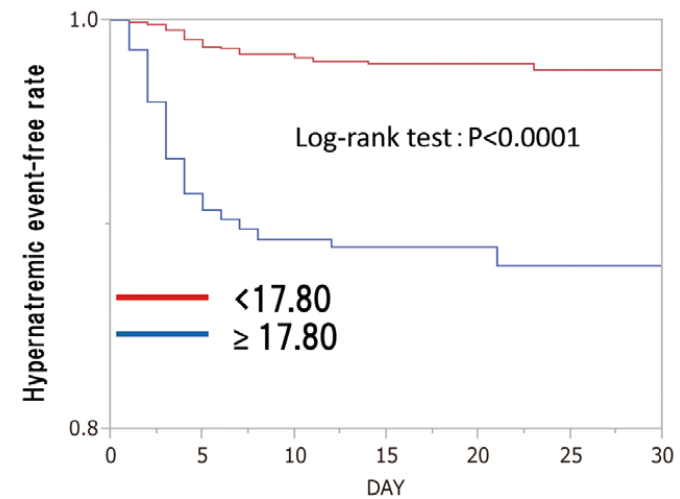

Figure 3. Risk score equation predicting hypernatremic events and (A) the distribution of risk scores and (B) Kaplan-Meier analysis using a risk score cut-off of 17.80. Na, serum sodium concentration; BUN, blood urea nitrogen concentration; $\mathrm{Cr}$, serum creatinine concentration; $\mathrm{K}$, serum potassium concentration.
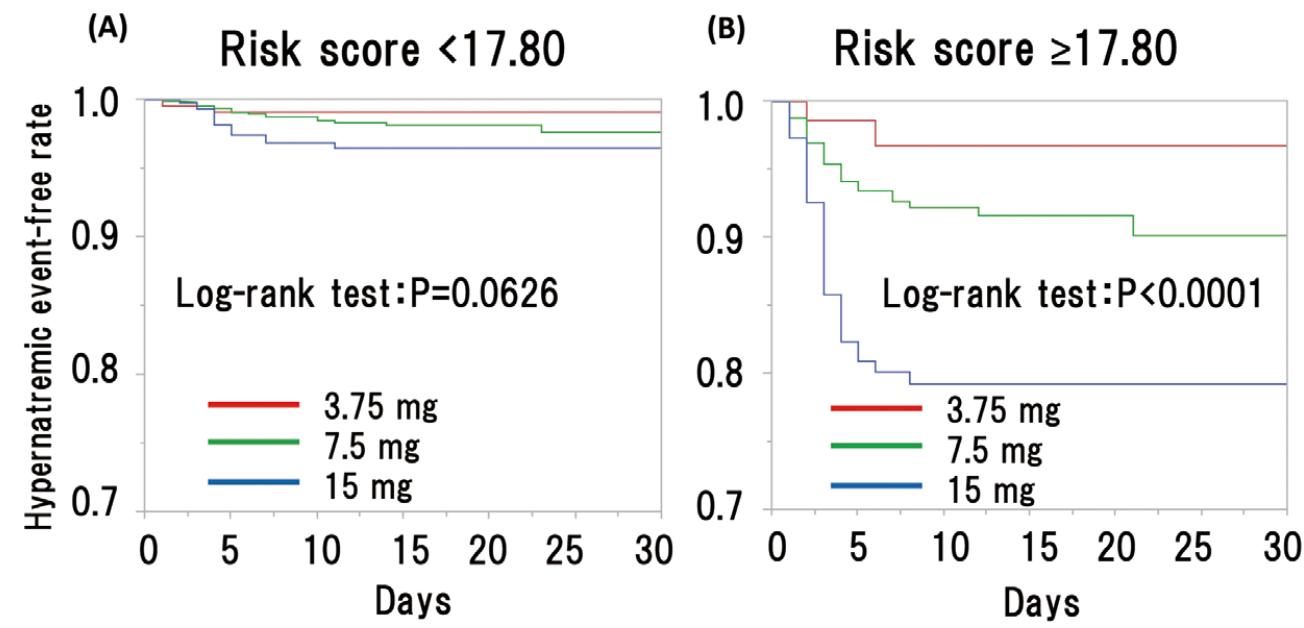

Figure 4. Kaplan-Meier analysis of the hypernatremic event-free rate stratified by risk score and the initial dose of tolvaptan.

7.5, and $15 \mathrm{mg}$ tolvaptan (Figure 4B). The model was evaluated by the c-index, and bootstrap resampling with 500 repetitions was used to calculate $95 \%$ CIs. The c-index of the risk model was 0.735 (95\% CI $0.660-0.805)$. A validation study was performed by randomly dividing the entire cohort $(\mathrm{N}=3,349)$ into a derivation cohort $(60 \%$; $\mathrm{n}=2,010)$ and a validation cohort $(40 \% ; \mathrm{n}=1,339)$. A c-index of the developed risk score of 0.761 was calculated for the derivation cohort and 0.703 for the validation cohort. In addition, entire cohort was divided 50/50 in a 
derivation and validation cohort, and the c-index was calculated as 0.752 for the derivation cohort $(n=1,675)$ and 0.721 for the validation cohort $(n=1,674)$. We confirmed that the c-index did not differ between the derivation and validation cohorts in these 2 validation studies.

Moreover, a calibration study was performed based on quintiles and deciles of the risk score. Table $\mathbf{S 2}$ lists the risk score and c-index for each decile and quintile point. We confirmed that our model was the best-fitted given that the c-index for each of the decile and quintile points was lower than in our model.

\section{Discussion}

In the present study we investigated the occurrence of hypernatremia, defined as serum sodium concentrations $\geq 150 \mathrm{mEq} / \mathrm{L}$, after tolvaptan treatment and identified risk factors that predicted adverse events. Moreover, we derived a novel 'risk score' from the ORs of risk factors to calculate the risk of hypernatremia before tolvaptan treatment and to allow us to identify the group of patients at high risk of hypernatremia.

In a retrospective study of 8,140 patients admitted to an intensive care unit, a mortality rate of $46.2 \%$ was reported for patients with hypernatremia. ${ }^{12}$ Another study reported increasing mortality risk ratios of 2.32 and 3.64 in patients with mild $(>150, \leq 155 \mathrm{mEq} / \mathrm{L})$ and severe $(>155 \mathrm{mEq} / \mathrm{L})$ hypernatremia. ${ }^{13}$ It is therefore essential to prevent the onset of hypernatremia in patients treated with tolvaptan, which may cause hypernatremia event because of its aquaretic effect. A simple and effective method to prevent this adverse drug reaction is desirable.

We have identified a set of risk factors that are predictive of hypernatremia. Based on the results of interim analyses of data from 1,053 patients, we reported that the risk factors for hypernatremia are as follows: baseline serum sodium concentrations $\geq 142 \mathrm{mEq} / \mathrm{L}$, serum potassium concentrations $<3.8 \mathrm{mEq} / \mathrm{L}$, and a higher initial tolvaptan dose of $15 \mathrm{mg} .{ }^{9}$ In the present final analysis, the BUN/Cr ratio was identified as a new risk factor for hypernatremia in addition to the 3 risk factors reported earlier. In fact, Hirai et al12 reported the following 3 risk factors for hypernatremia, defined as serum sodium concentrations $\geq 147 \mathrm{mEq} / \mathrm{L}$, in 371 patients with heart failure and liver cirrhosis: serum $\mathrm{Na}$ concentrations $\geq 140 \mathrm{mEq} / \mathrm{L}$, initial dose of tolvaptan $>7.5 \mathrm{mg}$, and BUN/Cr ratio $\geq 20$. Although the definition of hypernatremia Hirai et a $\mathbf{l}^{\mathbf{1 4}}$ used differed from that used in the present analysis, Hirai et $\mathrm{al}^{\mathbf{1 4}}$ also selected the BUN/ $\mathrm{Cr}$ ratio as a risk factor. The higher $\mathrm{BUN} / \mathrm{Cr}$ ratio may suggest a dehydrated state. Patients with dehydration must not be given tolvaptan. However, a high $\mathrm{BUN} / \mathrm{Cr}$ ratio does not necessarily mean dehydration is present, but may reflect a state of vasopressin activation. In fact, Matsue et al15 reported that tolvaptan improved the prognosis of acute heart failure patients using the combined endpoint of all-cause death and readmission in patients with a higher $\mathrm{BUN} / \mathrm{Cr}$ ratio of $>20$. If patients in heart failure with higher BUN/Cr ratios as a result of activated vasopressin signaling are a preferred target for the use of tolvaptan to improve prognosis, a safer treatment algorithm is required to continue tolvaptan treatment.

Serum potassium concentration was not found to be a risk factor in the study of Hirai et al. ${ }^{14}$ This difference could be attributed to the patient mix, because Hirai et a ${ }^{14}$ included patients with liver cirrhosis in their study. The etiology of volume overload is different in patients with liver cirrhosis. In fact, a lower incidence of hypernatremia $(0.2 \% ; 1 / 453)$ was reported in patients with liver cirrhosis treated with tolvaptan in the large-scale post-marketing surveillance START study. ${ }^{16}$ These results may suggest that aldosterone antagonists play a part of preventive effect of hypernatremia, because almost all patients in the study of Hirai et al ${ }^{14}$ with liver cirrhosis were administered spironolactone, which may increase serum potassium concentrations. In contrast, in the present study, the rate of use of aldosterone antagonists in heart failure was approximately $40 \%$. We hypothesize that aldosterone antagonists like spironolactone may prevent hypernatremic events by inhibiting aldosterone-induced sodium reabsorption. However, more detailed investigation is necessary to determine the role of aldosterone antagonists in preventing hypernatremic events.

As shown in Figure 1, tolvaptan did not increase the mean serum sodium concentration in patients with normonatremia. However, the equation we developed to calculate a risk score from the ORs suggests that the baseline sodium concentration is the most important risk factor for the development of hypernatremia. The need to use tolvaptan in patients whose baseline serum sodium concentrations are $\geq 145 \mathrm{mEq} / \mathrm{L}$ should be carefully determined in consideration of the balance between benefit and risk. We recommend calculating the risk score for each patient before starting treatment, and starting tolvaptan administration at the dose of $3.75 \mathrm{mg}$ in patients with a risk score $\geq 17.80$. Given that the efficacy of tolvaptan is expected to be low when administered at a dose of $3.75 \mathrm{mg}$, an increase in the dose to $7.5 \mathrm{mg}$ should be considered for patients who tolerate the drug. Recently (in June 2017), a new formulation of tolvaptan ( $1 \%$ oral granules) was launched in Japan. Physicians can now easily choose a lower dose depending on a patient's condition. Even with the lower dose of tolvaptan, it is still important that serum sodium concentrations are monitored in all patients who are treated with tolvaptan.

It is required that tolvaptan treatment is started in an inpatient setting. If the onset of hypernatremia can be prevented, tolvaptan may also be used in outpatient settings and may therefore become a more convenient therapy for patients. We recommend using the risk score to determine the optimal initial dose of tolvaptan to prevent the onset of hypernatremia.

\section{Study Limitations}

The present study was a naturalistic observational study conducted with a non-interventional cohort and did not aim to elucidate the characteristics of patients who report hypernatremic events while receiving tolvaptan. More accurate elucidation of risk factors for hypernatremic events will require further investigations with a study design suited to this purpose.

\section{Conclusions}

Risk factors for hypernatremia with the use of tolvaptan in real-world clinical settings were confirmed by analyzing data from the SMILE study. An equation was developed to calculate a risk score that predicts the risk of developing hypernatremia. We recommend that tolvaptan treatment be started at a lower dose in patients with a high $(\geq 17.80)$ risk score. 


\section{Acknowledgments}

The authors express their gratitude to the physicians across Japan who participated in this study. The authors also thank Mr. Makoto Takeda (A2 Healthcare Corporation) for the statistical analysis. The authors are grateful to Dr. Tomoo Funaki, Otsuka Pharmaceutical, for technical support in creating the nomogram. The nomogram to calculate the risk score is available at the following website: https:// riskscore.shinyapps.io/Nomogram/

\section{Name of Grant}

This study was supported by Otsuka Pharmacutical.

\section{Conflicts of Interest}

K.K., N.S., and T.I. have received honoraria from Otsuka Pharmaceutical for lectures.

\section{References}

1. Pearcock WF, Constanzo MR, De Marco T, Lopatin M, Wynne $\mathrm{J}$, Mills RM, et al. Impact of intravenous loop diuretics on outcome of patients hospitalized with acute decompensated heart failure: Insights from the ADHERE registry. Cardiology 2009; 113: $12-19$.

2. Zimily HD, Daifallah S, Ghali JK. Tolvaptan, hyponatremia, and heart failure. Int J Nephrol Renovasc Dis 2011; 4: 57-71.

3. Matsuzaki M, Hori M, Izumi T, Fukunami M; Tolvaptan Investigators. Efficacy and safety of tolvaptan in heart failure patients with volume overload despite the standard treatment with conventional diuretics: A Phase III, randomized, double-blind, placebo-controlled study (QUEST Study). Cardiovasc Drug Ther 2011; 25(Suppl 1): S33-S45.

4. Kinugawa K, Imamura T, Komuro I. Experience of a vasopressin receptor antagonist, tolvaptan, under the unique indication in Japanese heart failure patients. Clin Pharmacol Ther 2013; 94: $449-451$.

5. Yamamura $Y$, Nakamura $S$, Itoh $S$, Hirano $T$, Onogawa $T$, Yamashita T, et al. OPC-41061, a highly potent human vasopressin $\mathrm{V}_{2}$-receptor antagonist: Pharmacological profile and aquaretic effect by single and multiple oral dosing in rats. J Pharmacol Exp Ther 1998; 287: 860-867.

6. Konstam MA, Gheorghiade M, Burnett JC Jr, Grinfeld L, Maggioni AP, Swedberg K, et al. Effects of oral tolvaptan in patients hospitalized for worsening heart failure: The EVEREST Outcome Trial. JAMA 2007; 297: 1319-1331.

7. Gheorghiade M, Niazi I, Ouyang J, Czerwiec F, Kambayashi J, Zampino $\mathrm{M}$, et al. Vasopressin $\mathrm{V}_{2}$-receptor blockade with tolvaptan in patients with chronic heart failure: Results from a double-blind, randomized trial. Circulation 2003; 107: $2690-$ 2696.

8. Matsuzaki M, Hori M, Izumi T, Fukunami M; the Tolvaptan Investigators. Efficacy and safety of tolvaptan in heart failure patients with volume overload despite the standard treatment with conventional diuretics: A phase III, randomized, doubleblind, placebo-controlled Study (QUEST Study). Cardiovasc Drugs Ther 2011; 25: S33-S45.

9. Kinugawa K, Sato N, Inomata T, Shimakawa T, Iwatake N, Mizuguchi K. Efficacy and safety of tolvaptan in heart failure patients with volume overload. Circ J 2014; 78: 844-852.

10. Kinugawa K, Inomata T, Sato N, Yasuda M, Shimakawa T, Bando K, et al. Effectiveness and adverse events of tolvaptan in octogenarians with heart failure interim analyses of Samsca Post-Marketing Surveillance In Heart faiLurE (SMILE Study). Int Heart J 2015; 56: 137-143.

11. https://ctep.cancer.gov/protocoldevelopment/electronic applications/ctc.htm (accessed July 4, 2013).

12. Darmon M, Timsit JF, Francais A, Nquile-Makao M, Adrie C, Cohen Y, et al. Association between hypernatraemia acquired in the ICU and mortality: A cohort study. Nephrol Dial Transplant 2010; 25: 2510-2515.

13. Funk GC, Lindner G, Druml W, Metnitz B, Schwarz C, Bauer $\mathrm{P}$, et al. Incidence and prognosis of dysnatremias present on ICU admission. Intensive Care Med 2010; 36: 304-311.

14. Hirai K, Shimomura T, Moriwaki H, Ishii H, Shimoshikiryo T, Tsuji D, et al. Risk factors for hypernatremia in patients with short- and long-term tolvaptan treatment. Eur J Clin Pharmacol 2016; 72: $1177-1183$.

15. Matsue Y, Suzuki M, Torii S, Yamaguchi S, Fukamizu S, Ono $Y$, et al. Prognostic impact of early treatment with tolvaptan in patients with acute heart failure and renal dysfunction. Int $J$ Cardiol 2016; 221: 188-193.

16. Sakaida I, Terai S, Kurosaki M, Yasuda M, Okada M, Bando $\mathrm{K}$, et al. Effectiveness and safety of tolvaptan in liver cirrhosis patients with edema -interim results of Samsca posT-mARkeTing surveillance in liver cirrhosis (START study). Hepatol Res 2017; 47: $1137-1146$.

\section{Supplementary Files}

\section{Supplementary File 1}

Table S1. Summary of the 14 patients with serious hypernatremia reported by physicians

Table S2. Results of the calibration study: c-index based on quintiles and deciles of the risk score

Please find supplementary file(s);

http://dx.doi.org/10.1253/circj.CJ-17-0986 